\title{
Persistent risk of subsequent procedures and mortality in patients after interrupted aortic arch repair: A Congenital Heart Surgeons' Society study
}

\author{
Anusha Jegatheeswaran, Hon.BSc, MD, ${ }^{\mathrm{a}}$ Brian W. McCrindle, MD, MPH, ${ }^{\mathrm{b}}$ Eugene H. Blackstone, MD, \\ Marshall L. Jacobs, MD, ${ }^{\mathrm{d}}$ Gary K. Lofland, MD, ${ }^{\mathrm{e}}$ Earl H. Austin III, MD, ${ }^{\mathrm{f}}$ Thomas Yeh, MD, PhD, ${ }^{\mathrm{g}}$ \\ Victor Morell, MD, ${ }^{\mathrm{h}}$ Jeffrey P. Jacobs, MD, ${ }^{\mathrm{i}}$ Richard A. Jonas, MD, ${ }^{\mathrm{j}}$ Sally Cai, MSc, ${ }^{\mathrm{a}}$ \\ Jeevanantham Rajeswaran, MSc, ${ }^{\mathrm{c}}$ Marco Ricci, MD, ${ }^{\mathrm{k}}$ William G. Williams, MD, ${ }^{\mathrm{a}}$ \\ Christopher A. Caldarone, MD, ${ }^{a}$ and William M. DeCampli, MD, $\mathrm{PhD}^{1}$
}

Objective: Multiple subsequent procedures directed at the arch and/or the left ventricular outflow tract are frequently required after interrupted aortic arch repair. We the investigated patterns and factors associated with these subsequent procedures and mortality.

Methods: We reviewed the data from 447 patients with interrupted aortic arch at 33 institutions enrolled from 1987 to 1997 . We classified the subsequent procedures by type (catheter-based or surgical) and focus (arch, left ventricular outflow tract, and "other" cardiovascular lesions). We used competing risks and modulated renewal analysis to explore subsequent procedures.

Results: There were 158 subsequent arch and 100 left ventricular outflow tract procedures. Freedom from death at 21 years was $60 \%$ overall. The risk of additional subsequent arch procedures decreased after the first subsequent arch procedure in the acute phase, but did not significantly change in the chronic phase. The risk of additional subsequent left ventricular outflow tract procedures increased after the first subsequent left ventricular outflow tract procedure in the chronic phase. The risk factors for subsequent arch procedures and mortality, but not for subsequent outflow track procedures, were related in a complex way to previous procedures and their timing.

Conclusions: Interrupted aortic arch is a chronic disease in which patients often undergo multiple subsequent procedures with persistent risk for additional intervention and mortality. The risk factors are related to the nature and timing of previous procedures and to the morphology and details of the index procedure. Interrupted aortic arch should be considered a chronic disorder. (J Thorac Cardiovasc Surg 2010;140:1059-75)

Supplemental material is available online.

For survivors of neonatal repair of an interrupted aortic arch (IAA), subsequent procedures, particularly for aortic arch (arch) or left ventricular outflow tract (LVOT) obstruction, are common and are associated with mortality. One question is whether repeated subsequent procedures on the arch or LVOT are a continuing risk after repair of an IAA. Given the current excellent operative survival after the ini-

\footnotetext{
From the Division of Cardiovascular Surgery ${ }^{\mathrm{a}}$ and the Division of Cardiology, ${ }^{\mathrm{b}}$ Hospital for Sick Children, University of Toronto, Toronto, Ontario, Canada; the Heart and Vascular Institute and Quantitative Health Sciences, ${ }^{c}$ Cleveland Clinic Foundation, Cleveland, Ohio; Pediatric and Congenital Heart Surgery, ${ }^{\mathrm{d}}$ Cleveland Clinic, Cleveland, Ohio; the Department of Cardiothoracic Surgery, ${ }^{\mathrm{e}}$ Children's Mercy Hospital, Kansas, Mo; Division of Thoracic and Cardiovascular Surgery, ${ }^{\mathrm{f}}$ University of Louisville, Louisville, Ky; the Division of Cardiothoracic Surgery, ${ }^{\mathrm{g}}$ Tulane University School of Medicine, New Orleans, La; the Department of Cardiothoracic Surgery, ${ }^{\mathrm{h}}$ Children's Hospital of Pittsburgh, Pittsburgh, Pa; Division of Thoracic and Cardiovascular Surgery, ${ }^{\mathrm{i}}$ Department of Surgery, Congenital Heart Institute of Florida, All Children's Hospital and Children's Hospital of Tampa, Cardiac Surgical Associates of Florida, Saint Petersburg, Fla; the Division of Cardiac Surgery, ${ }^{\mathrm{j}}$ Center for Heart, Lung and Kidney Disease, Children's National Medical, Washington, DC; the Division of Cardiothoracic Surgery, ${ }^{\mathrm{k}}$ University of Miami
}

tial neonatal repair, this question emerges as one addresses the longer term morbidity and functional health status of patients with this infrequently encountered anomaly. Two previous studies by the Congenital Heart Surgeons' Society (CHSS) examined the outcomes of patients with IAA, but they did not address repeated or subsequent arch or LVOT procedures and their associated factors. ${ }^{1,2}$ This report builds on the previous 2005 CHSS report by adding 5 additional years of follow-up and adding evaluation of the time-related rates of, and associated factors for, subsequent arch and LVOT procedures after the index IAA repair, in addition to mortality. ${ }^{2}$

Health Systems, Miller School of Medicine, Miami, Fla; and the Division of Cardiothoracic Surgery, ${ }^{1}$ The Congenital Heart Institute at Arnold Palmer Hospital for Children and University of Central Florida College of Medicine, Orlando, Fla. Disclosures: None

Read at the 90th Annual Meeting of The American Association for Thoracic Surgery, Toronto, Ontario, Canada, May 1-5, 2010.

Received for publication May 4, 2010; accepted for publication July 30, 2010.

Address for reprints: William M. DeCampli, MD, PhD, Division of Cardiothoracic Surgery, The Congenital Heart Institute at Arnold Palmer Hospital for Children and University of Central Florida College of Medicine, 50 West Sturtevant St, Orlando, FL 32806 (E-mail: william.decampli@orlandohealth.com). $0022-5223 / \$ 36.00$

Copyright (๑) 2010 by The American Association for Thoracic Surgery doi:10.1016/j.jtcvs.2010.07.067 


\section{Abbreviations and Acronyms \\ CHSS $=$ Congenital Heart Surgeons Society \\ IAA $=$ interrupted aortic arch \\ LVOT $=$ left ventricular outflow tract \\ VSD $=$ ventricular septal defect}

\section{MATERIALS AND METHODS}

Between January 1987 and December 1997, 472 neonates with IAA admitted within 30 days of birth were enrolled by 33 CHSS member institutions (Appendix 1). IAA was defined as either a complete discontinuity or a nonpatent fibrous strand in the transverse arch or aortic isthmus, as described in the operative report. The 25 patients who did not undergo arch repair were excluded, leaving 447 patients in the study. The "index procedure" was defined as the initial procedure, consisting of repair of the arch discontinuity with or without simultaneous repair of the ventricular septal defect (VSD), LVOT obstruction, or other cardiovascular anomalies ("other"). A "subsequent procedure" was defined as one that occurred after the index procedure. The characteristics of the patients and cardiac morphology are summarized in Table $1, A$. Institutional and patient participation was voluntary and confidential. The patients provided informed consent, and approval was obtained according to the local requirements. Ethics approval for the CHSS Data Center was obtained annually from the Research Ethics Board of the Hospital for Sick Children, Toronto, Ontario, Canada.

\section{Data Collection}

The data were abstracted from copies of de-identified medical records submitted to the CHSS Data Center, as described previously. ${ }^{2}$ The variables recorded have been defined and described in our previous work. ${ }^{2}$ The most recent cross-sectional follow-up was performed between January and October 2008. Of the 447 patients, 169 had died and 278 were living. Follow-up was complete for $320(72 \%)$ of 447 patients. The median follow-up was 13.5 years (range, 13 days to 21.4 years) for surviving patients.

\section{Statistical Analysis}

The goals of the analysis were to describe (1) the spectrum, frequency, and timing of subsequent arch and LVOT procedures, (2) the time-related occurrence of mutually exclusive outcomes after a first or second subsequent arch or LVOT procedure using a nested competing risks methodology, (3) the time-related probability of repeated subsequent arch and LVOT procedures using a modulated renewal methodology that incorporated or adjusted for all procedures as time-varying covariates, and (4) the factors associated with subsequent arch procedures, LVOT procedures, and mortality. Both the nested competing risks and modulated renewal methods used multiphase parametric modeling of the hazard function, as previously described. ${ }^{3}$ Data are expressed as the frequency, median with the range, or mean and standard deviation, with the number of missing values indicated. All analyses were performed using Statistical Analysis Systems software, version 9.2 (SAS Institute, Inc, Cary, NC). The statistical methods are described in greater detail in Appendix 2.

\section{RESULTS}

\section{Overall Status After Index Repair}

The characteristics of the index repair are described in Table $1, B$. Of the 447 patients undergoing index IAA repair, 44 had their first LVOT procedure at the index repair. Of 447 patients undergoing index repair, 133 had died with no subsequent arch or LVOT procedure (21 having undergone some "other" procedure), and 154 patients were alive at the most recent follow-up, with no subsequent arch or LVOT procedures. Fifty of these 154 patients had undergone an "other" procedure. A total of 160 patients have had one or more subsequent arch and/or LVOT procedures, with or without "other" procedures. Of these, 119 patients had 158 subsequent arch procedures and 69 patients had 100 subsequent LVOT procedures (not mutually exclusive). Of these 160 patients, $36(23 \%)$ were alive. A display of cumulative risk of subsequent procedures over time is shown in Figure E1, illustrating not only a high risk of early procedures after index repair but also a continuing non-zero rate of subsequent procedures 1 to 2 decades after repair. The most common subsequent arch procedures were transcatheter balloon dilations and surgical patch augmentation (Appendix 3,A). The most common subsequent LVOT procedures were fibromuscular resection, the Konno procedure, and transcatheter balloon dilation (Appendix 3,B). Appendix 3, $A-D$, list the types of procedures stratified by the subsequent procedure number.

\section{Competing Risks for First and Second Subsequent Arch Procedures and Death}

Competing risks showed that 15 years after the index repair, $32 \%$ had died without a first subsequent arch procedure, $29 \%$ had undergone a first subsequent arch procedure, and 39\% remained alive without a first subsequent arch procedure (Figure 1,A). Of those patients who had undergone a first subsequent arch procedure; 15 years later, $22 \%$ had died without a second subsequent arch procedure, $31 \%$ had undergone a second subsequent arch procedure, and $47 \%$ remained alive without a second subsequent arch procedure (Figure 1, B).

\section{Competing Risks for First and Second Subsequent LVOT Procedures and Death}

Competing risks showed that 15 years after the index repair, 33\% had died without a first subsequent LVOT procedure, $18 \%$ had undergone a first subsequent LVOT procedure and remained at risk, $1 \%$ had undergone a first subsequent LVOT procedure and were no longer at risk of additional procedures, and $48 \%$ remained alive without a first subsequent LVOT procedure (Figure 2, A). For those patients having a first subsequent LVOT procedure, 15 years later, $13 \%$ had died without a second subsequent LVOT procedure, $44 \%$ had undergone a second subsequent LVOT procedure, and $43 \%$ remained alive without a second subsequent LVOT procedure (Figure 2, B).

\section{Subsequent Arch Procedures and Their Associated Factors}

The overall hazard function for any subsequent arch procedures showed 2 phases, an early or acute phase, accounting for 102 events, and an ongoing or chronic phase accounting for 56 events. Stratification of the overall hazard function into each subsequent arch procedure (first, second, third, 
TABLE 1. Patient characteristics and characteristics of index aortic arch repair

\begin{tabular}{|c|c|c|}
\hline Variable & No. (missing) & Value \\
\hline \multicolumn{3}{|l|}{ A. Patient characteristic } \\
\hline \multicolumn{3}{|l|}{ Demographic characteristics } \\
\hline Age at admission $(\mathrm{d}$, mean $\pm \mathrm{SD})$ & $447(0)$ & $4.41 \pm 5.28$ \\
\hline Birth weight $(\mathrm{kg}$, mean $\pm \mathrm{SD})$ & $198(249)$ & $2.55 \pm 1.29$ \\
\hline Gender (female/male) & $222 / 225$ & $50 \% / 50 \%$ \\
\hline Noncardiac anomaly & $155(0)$ & $35 \%$ \\
\hline DiGeorge syndrome & $81(0)$ & $18 \%$ \\
\hline \multicolumn{3}{|l|}{ Morphologic characteristics } \\
\hline Type of IAA & $446(1)$ & \\
\hline Type A & 125 & $28 \%$ \\
\hline Type B & 318 & $71 \%$ \\
\hline Type C & 3 & $1 \%$ \\
\hline Major associated cardiac anomalies & $447(0)$ & \\
\hline None (with isolated VSD) & 326 & $73 \%$ \\
\hline None (with no VSD) & 6 & $1 \%$ \\
\hline Aortopulmonary window & 19 & $4 \%$ \\
\hline $\begin{array}{l}\text { Complete atrioventricular septal } \\
\text { defect }\end{array}$ & 3 & $1 \%$ \\
\hline Atrioventricular discordance & 2 & $.4 \%$ \\
\hline Double-outlet right ventricle & 8 & $2 \%$ \\
\hline $\begin{array}{l}\text { Partial anomalous pulmonary } \\
\text { venous drainage }\end{array}$ & 1 & $.2 \%$ \\
\hline Single ventricle & 13 & $3 \%$ \\
\hline Taussig-Bing & 5 & $.1 \%$ \\
\hline $\begin{array}{l}\text { Transposition of great arteries } \\
\text { with VSD }\end{array}$ & 20 & $4 \%$ \\
\hline Truncus arteriosus & 45 & $10 \%$ \\
\hline Bicuspid aortic valve & $143(230)$ & $66 \%$ \\
\hline Anomalous right subclavian artery & $103(42)$ & $25 \%$ \\
\hline Left superior vena cava & $33(31)$ & $8 \%$ \\
\hline Large patent ductus arteriosus & $239(174)$ & $88 \%$ \\
\hline Large VSD & $308(71)$ & $82 \%$ \\
\hline Multiple VSDs & $29(84)$ & $8 \%$ \\
\hline Malalignment of VSD & $221(0)$ & $49 \%$ \\
\hline \multicolumn{3}{|l|}{ B. Characteristic of IAA repair } \\
\hline \multicolumn{3}{|l|}{ Demographic characteristics } \\
\hline Age at operation $(\mathrm{d}$, mean $\pm \mathrm{SD})$ & $447(0)$ & $9.81 \pm 19.74$ \\
\hline $\begin{array}{l}\text { Weight at index IAA repair } \\
\quad(\mathrm{kg}, \text { mean } \pm \mathrm{SD})\end{array}$ & $361(86)$ & $3.18 \pm 0.86$ \\
\hline \multicolumn{3}{|l|}{ Technique of arch repair } \\
\hline Approach & $447(0)$ & \\
\hline Median sternotomy & 323 & $72 \%$ \\
\hline Thoracotomy & 122 & $27 \%$ \\
\hline Both sternotomy and thoracotomy & 2 & $.4 \%$ \\
\hline Augmentation of aortic arch & $114(0)$ & $26 \%$ \\
\hline Type of IAA repair & $447(0)$ & \\
\hline Direct anastomosis with no patching & 265 & $59 \%$ \\
\hline Direct anastomosis with patching & 122 & $27 \%$ \\
\hline Interposition graft & 58 & $13 \%$ \\
\hline $\begin{array}{l}\text { Main pulmonary artery-aorta bypass } \\
\text { conduit }\end{array}$ & 2 & $.4 \%$ \\
\hline Use of graft material & $447(0)$ & \\
\hline Polytetrafluoroethylene & 57 & $13 \%$ \\
\hline Pulmonary artery homograft & 52 & $12 \%$ \\
\hline
\end{tabular}

TABLE 1. Continued

\begin{tabular}{lcc}
\hline \multicolumn{1}{c}{ Variable } & No. (missing) & Value \\
\hline Other & 28 & $6 \%$ \\
Pericardium & 23 & $5 \%$ \\
Aortic homograft & 16 & $4 \%$ \\
Xenograft & 4 & $1 \%$ \\
Unspecified homograft & 3 & $1 \%$ \\
Subclavian artery & $447(0)$ & \\
None & 385 & $86 \%$ \\
Left divided & 39 & $9 \%$ \\
Right divided & 23 & $5 \%$ \\
Both divided & 6 & $1 \%$ \\
\hline
\end{tabular}

Data are presented as numbers (\%) or mean \pm standard deviation.
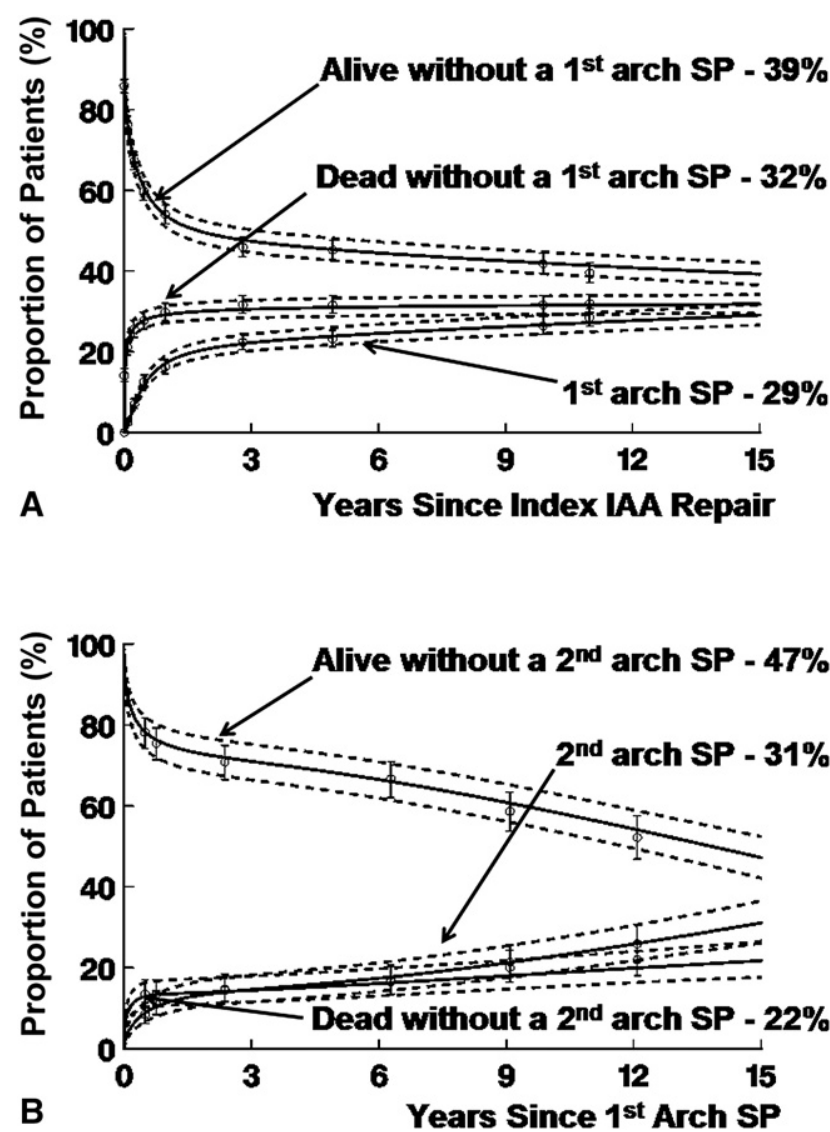

FIGURE 1. Competing risks for first and second subsequent aortic arch procedures. A, All patients started at index interrupted aortic arch (IAA) repair $(n=447)$ and could transition to either subsequent aortic arch procedure for residual or recurrent obstruction at arch repair site or death. B, All patients began at time of first subsequent aortic arch procedure $(\mathrm{n}=119)$ and could transition to either subsequent aortic arch procedure for residual or recurrent obstruction at arch repair site or death. Solid lines represent parametric point estimates; dashed lines enclose $70 \%$ confidence intervals; circles with error bars represent nonparametric estimates. Proportion of patients (expressed as a percentage of total) in each category at any given time. Arch, aortic arch; SP, subsequent procedure. 

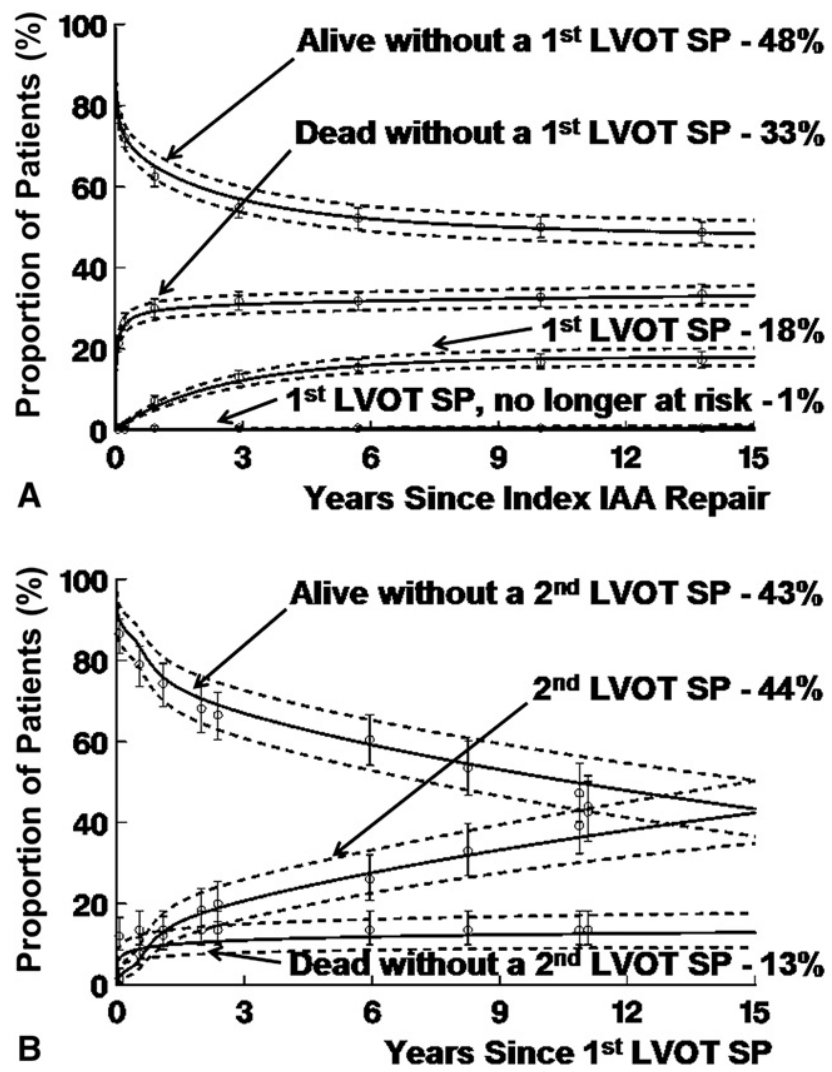

FIGURE 2. Competing risks for first and second subsequent left ventricular outflow tract (LVOT) procedures. A, All patients began at index interrupted aortic arch $(I A A)$ repair $(\mathrm{n}=423)$ and could transition to either subsequent LVOT procedure (still at risk or no longer at risk of additional LVOT procedures) for residual or recurrent obstruction at LVOT or death. B, All patients began at time of first subsequent LVOT procedure $(n=67)$ and could transition to either subsequent LVOT procedure for residual or recurrent obstruction at LVOT or death. Patients considered no longer at risk of LVOT procedures underwent repairs such as the Damus-KayeStansel procedure or heart transplantation and were censored at that point. Solid lines represent parametric point estimates; dashed lines enclose $70 \%$ confidence intervals; circles with error bars represent nonparametric estimates. $Y$-axis, Proportion of patients (expressed as percentage of total) in each category at any given point. $S P$, subsequent procedure.

and so forth) showed that the acute phase risk decreased between the first and second subsequent procedures and showed a trend for the lowest risk for the third subsequent procedure. The chronic phases showed no statistically significant change in the risk with subsequent arch procedures (Figure 3, A). The final multivariate model is shown in Appendix 4. Associations that significantly increased risk of any subsequent arch procedure in the early ("acute") and late ("chronic") hazard phases are listed in Table 2.

\section{Subsequent LVOT Procedures and Their Associated Factors}

The overall hazard function for any subsequent LVOT procedures showed 2 phases, an early or "acute" phase
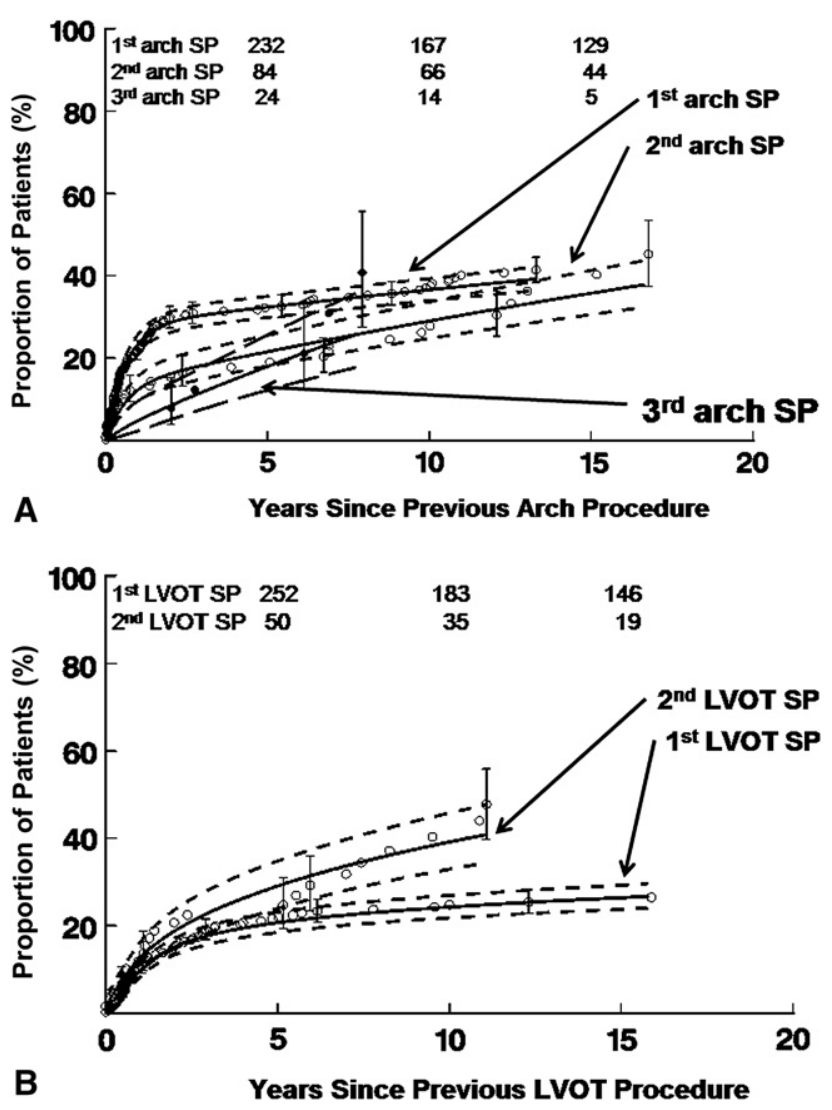

FIGURE 3. Modulated renewal for subsequent aortic arch and left ventricular outflow tract $(L V O T)$ procedures. A, All patients began at previous arch procedure $(\mathrm{n}=447)$. B, All patients began at previous LVOT procedure $(\mathrm{n}=423)$. Solid lines represent parametric point estimates; dashed lines enclose $70 \%$ confidence intervals; circles represent events. Each curve represents number of patients undergoing successive repair. Each curve truncated at last event. Proportion of patients at risk expressed as percentages. Number of patients alive and at risk at 5, 10, and 15 years for each renewal listed across top of graph. Patients considered no longer at risk of LVOT procedures, who underwent repairs such as Damus-KayeStansel or heart transplantation, were censored at that point. Arch, aortic arch; $S P$, subsequent procedure.

accounting for 50 events, and an ongoing or "chronic" phase accounting for 50 events. Stratification of the overall hazard function into each subsequent LVOT procedure (first, second, third, and so forth) showed that the early phase risk increased slightly between the first and second subsequent procedures, although the difference was not statistically significant. The ongoing or chronic phase risk of a second subsequent LVOT procedure was significantly greater than that for a first subsequent procedure (Figure 3, B). The final multivariate model is shown in Appendix 4. Associations that were significantly associated with an increased risk of subsequent LVOT procedures are listed in Table 2. Although the risk was increased when the immediately preceding procedure was the index 
TABLE 2. Associations that increase risk

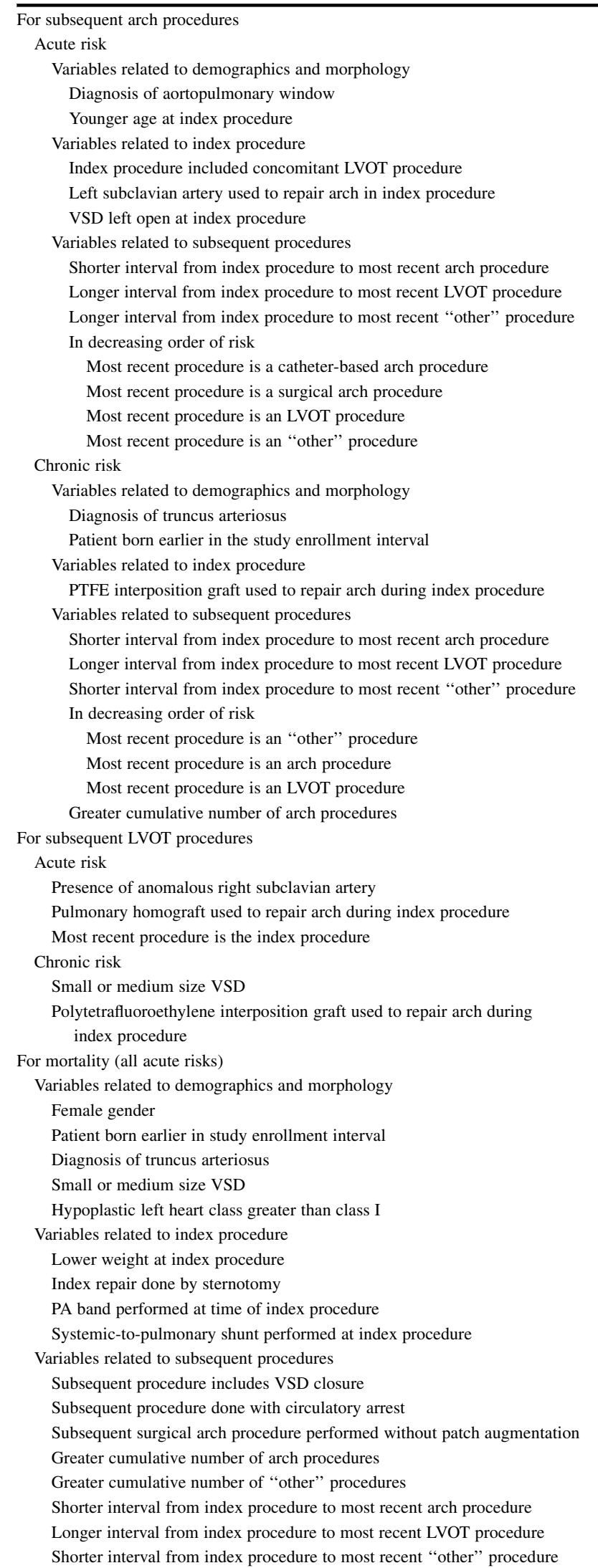

procedure, the presence of an immediately preceding subsequent LVOT procedure was not a risk factor. This finding is in contrast to that for subsequent arch procedures (see above).

\section{Mortality and Its Associated Factors}

Of the 447 patients, 169 have died. The hazard function for time-related mortality was characterized by a more prolonged early phase only, with survival at 21 years of $60 \%$ (70\% confidence interval, 57\%-62\%) (Appendix 5). The final multivariate model is shown in Appendix 4. Associations that significantly increased the risk of mortality are listed in Table 2.

\section{DISCUSSION \\ Previous studies}

Past studies have reported widely ranging estimates of survival for patients with IAA, with more recent studies reporting improvements. These include $47 \%$ at 10 years $\left(\mathrm{n}=63\right.$, dates of operation 1974-1987), ${ }^{4} 85 \%$ at 12 years $(\mathrm{n}=72$, dates of operation $1985-1997),{ }^{5} 70 \%$ at 5 years $\left(\mathrm{n}=82\right.$, dates of operation 1985-1995), ${ }^{6} 67 \%$ at 10 years $\left(n=94\right.$, dates of operation 1975-1999) ${ }^{7}$ $50 \%$ at 30 days $(\mathrm{n}=40$, dates of operation 19771997). ${ }^{8}$ Although initially a staged approach was thought to produce better outcomes, ${ }^{9-11}$ primary repair is now the favored approach, ${ }^{12-14}$ with selective use of a staged repair. ${ }^{8}$

Our previous 2005 CHSS study demonstrated that subsequent arch and LVOT procedures are common after IAA repair. Additionally, we found that (1) patients with a low birth weight, immediate presentation, type B IAA, and major associated cardiac anomalies remained at increased risk of death and initial LVOT procedure, (2) index arch repair using direct anastomosis with nonpolytetrafluroethylene patch augmentation was associated with reduced mortality, (3) patients whose index operation included an LVOT procedure were at a greater risk of death and more complex subsequent management, and (4) LVOT obstruction managed with catheter-based techniques was associated with increased recurrence rates and the need for an additional subsequent procedure.

Recent studies have corroborated our previous and current findings that subsequent arch and LVOT procedures are common after IAA repair. In a study of 65 patients with 55 early survivors, Brown and colleagues ${ }^{15}$ found that 20 patients underwent 27 reoperations between 1 week and 9 years postoperatively; 15 patients had a subsequent arch procedure, 13 surgical and 2 catheter-based. The 15-year actuarial freedom from subsequent arch, LVOT, or any type of procedure was $74 \%, 92 \%$, and $60 \%$, respectively. They could not identify any factors associated with subsequent procedures. Hussein and colleagues ${ }^{16}$ studied 112 patients with IAA undergoing the 
index repair between 1985 and 2007. There were 11 early deaths, and 12 early and 19 late subsequent arch procedures. An additional 16 patients had significant arch obstruction at the time of the study. The factors associated with subsequent arch procedure were the index repair technique other than direct anastomosis and the need for subsequent LVOT procedure. Tlaskal and colleagues ${ }^{17}$ studied 50 patients undergoing IAA repair using direct arch anastomosis between 1990 and 2009. Of the 40 early survivors, 17 required subsequent procedures. Mishra ${ }^{18}$ recently reviewed the extant published data on IAA. None of these reports focused on the risk factors for subsequent procedures after the first intervention.

\section{Present Study}

The present study focused on estimating the hazard for subsequent arch and LVOT procedures (ie, after the index procedure). In the present study (in contrast to the previous CHSS work), we added the use of a statistical technique, modulated renewal with adjustment for time-varying covariates, to examine the inter-relationships of such procedures. In this renewal model, the baseline hazard function for a subsequent procedure was assumed to be dependent only on the time since the nearest previous procedure of its kind, modulated by other risk factors that might be dependent on the time since the index repair. The classic analogy is that of a refrigerator, which usually fails because its compressor motor fails. The risk of failure depends, mostly, only on the interval since the most recent motor replacement ("subsequent procedure"), rather than on the interval since the original motor was installed ("index procedure"). Additionally, other characteristics of the refrigerator ("anatomic factors") or how it is repaired ("procedures") might contribute to the risk, some of those appearing between motor changes and "modulating" the renewal.

\section{Principle Findings}

Our first finding was that multiple procedures after index repair are common. As such, IAA is often a chronic disorder and not a structural anomaly definitively treated by a single operation in the newborn period. Of the 447 index procedures, the cohort experienced 158 subsequent arch procedures, 100 subsequent LVOT procedures, and 192 subsequent "other" procedures. Many patients underwent multiple subsequent procedures, with 2 patients each having undergone 11.

Our second finding was that although the acute risk of subsequent arch procedures decreased after each subsequent arch procedure, the chronic risk showed no significant trend. Moreover, the acute risk of subsequent LVOT procedure showed no significant trend, and the chronic risk increased after each subsequent LVOT procedure. This finding underscores the chronicity of the disorder, because the hazards show no long-term tendency to decrease. The different patterns we found between the subsequent arch and LVOT procedure hazards might reflect the differences in how the arch and LVOT respond to subsequent procedures. The arch, for example, will normally grow, except perhaps in discrete areas of recurrent stenosis. Subsequent procedures directed at the more discrete areas will generally be long lasting in the older child, and the hazard will plateau. In contrast, certain LVOT anatomic configurations have a propensity for recurrence (as in the subaortic membrane), and local resection or patching might be inadequate for long-term relief or might actually stimulate fibromuscular proliferation. In addition, LVOT obstruction might be multilevel (supravalvar, valvar, subvalvar discrete or tunnel-like), with different levels becoming significantly obstructive at different times. Such a trend has been demonstrated after a variety of operations associated with the risk of LVOT obstruction. ${ }^{19}$

Our third finding was that factors associated with subsequent arch procedure were related to previous procedures, as well as to characteristics of the anatomy and the index repair. Most of the anatomic factors and factors related to the index repair have been found to increase risk in previous studies. ${ }^{18}$ The present study is the first to demonstrate risk factors related to previous procedures (Table 2). At any time " $t$," the likelihood of a subsequent arch intervention was greatest when the most recent procedure was a catheter-based arch procedure, followed (in descending order of magnitude of risk) by surgical arch, LVOT, and "other" procedure in the acute phase. These risks were also present in the chronic phase, although in somewhat different order (see Figure E2). This implies that, at least in the era under consideration, catheter-based arch intervention might be less durable than surgical intervention for arch obstruction. The most recent procedure being an arch procedure was also a significant risk factor. This might be accounted for by patients with more complicated arch problems, with increased chance of failure of a previous attempt at correction (particularly at the index procedure). This rationale is further supported because the shorter interval from the index procedure to the most recent arch procedure is also a risk factor, the shorter interval reflecting the inadequacy of repair, the limited tissue growth within that short interval, or the complexity of the arch pathology (Figure E2). Other factors associated with a chronic risk of subsequent arch procedure were similar to those associated with acute risk, with the addition of greater cumulative number of arch procedures. The latter risk factor, again, most likely reflects the complexity of the residual arch problem. As in the acute phase, in the chronic phase, the most recent procedure being an "other" procedure was a risk factor. This finding might reflect nothing more than the relative prevalence of "other" procedures (eg, conduit changes or staged operations) in the chronic phase. 
In contrast to subsequent arch procedures, we could not identify the risk factors for subsequent LVOT procedures that were related to previous procedures. This is perhaps due to the heterogeneous morphology of LVOT obstruction, institutional variability in the indications for reoperation, or the tendency to take a stepwise approach to potentially complex LVOT obstruction. In contrast, we found anatomic and index procedural risk factors to be commensurate with those of previous studies, and the associated factors included anomalous right subclavian artery, a small or medium VSD, and the use of a polytetrafluroethylene graft to repair the arch at index repair. That patch augmentation of the arch at index repair is an association might be owing to the necessity to patch a hypoplastic arch, which, in turn, was associated (pathophysiologically or morphologically) with LVOT obstruction. The immediate preceding procedure, being the index procedure, might be a risk factor because of the relatively high prevalence of "borderline" LVOTs that were left unrepaired at the index operation, which then required repair as the next procedure. Only 44 patients $(10 \%)$ underwent an LVOT procedure at the index repair, but 100 more LVOT procedures were subsequently performed. In contrast, LVOT repair at the index repair might not reduce the risk of subsequent LVOT procedure. In the series by Morales and colleagues, ${ }^{20}$ for example, $43 \%$ of patients underwent LVOT repair at the index operation, but the 5-year freedom from a subsequent LVOT procedure was only $66 \%$.

Our fourth finding was that subsequent procedures adversely affected survival. This effect might have been due to the procedure itself or to the clinical conditions that necessitated the procedure. This is illustrated by the associations that increased the risk for death listed in Table 2. The procedural risk factors included a subsequent procedure that involved VSD closure, circulatory arrest, or an arch procedure done without patch augmentation. The risk factors related to the timing and interaction of the subsequent procedures included a greater cumulative number of arch or "other" procedures, a shorter interval between the index repair and the most recent arch or "other" procedure, and a longer interval between the index repair and the closest preceding LVOT procedure. Most of these risk factors have plausible explanations. Subsequent VSD closure indicates a staged approach to repair that might be associated with increased mortality. Other than excision and primary anastomosis, arch procedures done without patch augmentation might be more palliative procedures, such as left ventricular to descending aortic bypass, interposition tube graft placement, or balloon dilatation, all of which could increase the mortality hazard. The cumulative number of arch or "other" procedures being a risk factor is consistent with each successive arch or "other" procedure being associated with an early phase risk of mortality, adding to the cumulative risk. The short interval between the index repair and the closest preceding arch or "other" procedure, as stated previously, indicates the rapidity of recurrence and/or the severity of residual lesions, which might be the most challenging and thus associated with greater risk operations or subsequent procedures. The relationship between mortality risk and the longer interval between the index repair and the closest preceding LVOT procedure might reflect delays in operative relief of recurrent LVOT obstruction, with resulting left ventricular dysfunction and greater mortality risk. None of these risk factors related to subsequent procedures has been previously elucidated. Our ability to identify them was a result of the large cohort, the long duration of follow-up, and the use of the statistical technique of modulated renewal.

Of particular interest was that patch augmentation, as a part of the index arch repair, was not associated with improved survival, although it was in the previous CHSS analysis. Although this was found to be a salutary factor in some studies, ${ }^{2,15}$ in other studies, direct anastomosis without a patch conferred better survival. ${ }^{16}$ Morales and colleagues $^{20}$ reported excellent results with direct anastomosis in a series of 60 patients, but that study did not include a comparison group (with patch augmentation). In the present study, patch augmentation of the arch at the index operation was associated with a subsequent LVOT procedure (see above), which indirectly might have indicated a greater mortality risk and thus neutralized the advantage of patch augmentation. In our view, the complex interplay of these risk factors leaves the question of the advantage of patch augmentation unanswered.

\section{Study Limitations}

The present study had several important limitations. First, because this was an observational inception study, we were unable to serially and consistently measure the morphologic characteristics (eg, LVOT diameter) that might have helped us to explain the associations among subsequent procedures that we observed. Second, enrollment by participating institutions was voluntary, allowing for the possibility of selection bias. Third, the enrollment period (1987-1997), although it afforded impressive longterm follow-up, represented an "early era" in the techniques of the index repair. Outcomes have significantly improved in the more recent era. For example, Morales and colleagues, ${ }^{20}$ examining a cohort undergoing repair between 1995 and 2005, reported 100\% freedom from a subsequent arch procedure at 5 years. In the latter study, it will be interesting to determine how the hazard for subsequent arch and LVOT procedures develops beyond a decade of follow-up. Fourth, our study focused on an analysis of subsequent procedures rather than on variables measuring the evolving pathologic features and pathophysiology (which might have helped us explain the pattern of subsequent procedures). As with the morphologic data, the latter would require a prospective study designed with the intent to measure these variables. 


\section{CONCLUSIONS}

Patients undergoing IAA repair are at persistent risk of subsequent procedures and mortality. Complex interrelationships exist among these subsequent procedures. IAA is a chronic disorder and not a structural anomaly definitively treated in the newborn period, a message that should be made clear to practitioners, patients, and their families alike.

We thank all the participating member institutions listed in Appendix 1, because, without their data, the present study would not have been possible. We also acknowledge the assistance of personnel from the CHSS Data Center, in particular, Olga Levesque and Maulik Baxi, for coordinating the collection and management of these data.

\section{References}

1. Jonas RA, Quaegebeur JM, Kirklin JW, Blackstone EH, Daicoff G. Outcomes in patients with interrupted aortic arch and ventricular septal defect: A multiinstitutional study. Congenital Heart Surgeons Society. J Thorac Cardiovasc Surg. 1994;107:1099-113.

2. McCrindle BW, Tchervenkov CI, Konstantinov IE, Williams WG, Neirotti RA, Jacobs ML, Blackstone EH. Risk factors associated with mortality and interventions in 472 neonates with interrupted aortic arch: A Congenital Heart Surgeons Society study. J Thorac Cardiovasc Surg. 2005;129:343-50.

3. Blackstone EH, Naftel DC, Turner MEJ. The decomposition of time-varying hazard into phases, each incorporating a separate stream of concomitant information. J Am Stat Assoc. 1986;81:615-24.

4. Sell JE, Jonas RA, Mayer JE, Blackstone EH, Kirklin JW, Castaneda AR. The results of a surgical program for interrupted aortic arch. $J$ Thorac Cardiovasc Surg. 1988;96:864-77.

5. Fulton JO, Mas C, Brizard CP, Cochrane AD, Karl TR. Does left ventricular outflow tract obstruction influence outcome of interrupted aortic arch repair? Ann Thorac Surg. 1999;67:177-81.

6. Serraf A, Lacour-Gayet F, Robotin M, Bruniaux J, Sousa-Uva M, Roussin R, Planche C. Repair of interrupted aortic arch: A ten-year experience. J Thorac Cardiovasc Surg. 1996;112:1150-60.

7. Schreiber C, Eicken A, Vogt M, Gunther T, Wottke M, Thielmann M, Paek SU, Meisner H, Hess J, Lange R. Repair of interrupted aortic arch: Results after more than 20 years. Ann Thorac Surg. 2000;70:1896-900.

8. Tlaskal T, Hucin B, Hruda J, Marek J, Chaloupecky V, Kostelka M, Janousek J, Skovranek J. Results of primary and two-stage repair of interrupted aortic arch. Eur J Cardiothorac Surg. 1998;14:235-42.

9. Mainwaring RD, Lamberti JJ. Mid- to long-term results of the two-stage approach for type B interrupted aortic arch and ventricular septal defect. Ann Thorac Surg. 1997;64:1782-6.

10. Irwin ED, Braunlin EA, Foker JE. Staged repair of interrupted aortic arch and ventricular septal defect in infancy. Ann Thorac Surg. 1991;52:632-9.

11. Qureshi SA, Maruszewski B, McKay R, Arnold R, West CA, Hamilton DI. Determinants of survival following repair of interrupted aortic arch in infancy. Int J Cardiol. 1990;26:303-12.

12. Luciani GB, Ackerman RJ, Chang AC, Wells WJ, Starnes VA. One-stage repair of interrupted aortic arch, ventricular septal defect, and subaortic obstruction in the neonate: A novel approach. J Thorac Cardiovasc Surg. 1996;111:348-58.

13. Vouhe PR, Mace L, Vernant F, Jayais P, Pouard P, Mauriat P, Leca F, Neveux JY. Primary definitive repair of interrupted aortic arch with ventricular septal defect. Eur J Cardiothorac Surg. 1990;4:365-70.

14. Tlaskal T, Chaloupecky V, Marek J, Hucin B, Kostelka M, Tax P, et al. Primary repair of interrupted aortic arch and associated heart lesions in newborns. J Cardiovasc Surg (Torino). 1997;38:113-8.

15. Brown JW, Ruzmetov M, Okada Y, Vijay P, Rodefeld MD, Turrentine MW. Outcomes in patients with interrupted aortic arch and associated anomalies: A 20year experience. Eur J Cardiothorac Surg. 2006;29:666-73. 664.

16. Hussein A, Iyengar AJ, Jones B, Donath SM, Konstantinov IE, Grigg LE, et al. Twenty-three years of single-stage end-to-side anastomosis repair of interrupted aortic arches. J Thorac Cardiovasc Surg. 2010;139:942-9.
17. Tlaskal T, Vojtovic P, Reich O, Hucin B, Gebauer R, Kucera V. Improved results after the primary repair of interrupted aortic arch: Impact of a new management protocol with isolated cerebral perfusion. Eur J Cardiothorac Surg. 2010;38: $52-8$.

18. Mishra PK. Management strategies for interrupted aortic arch with associated anomalies. Eur J Cardiothorac Surg. 2009;35:569-76.

19. Kalfa D, Ghez O, Kreitmann B, Metras D. Secondary subaortic stenosis in heart defects without any initial subaortic obstruction: a multifactorial postoperative event. Eur J Cardiothorac Surg. 2007;32:582-7.

20. Morales DL, Scully PT, Braud BE, Booth JH, Graves DE, Heinle JS, McKenzie ED, Fraser CD Jr. Interrupted aortic arch repair: Aortic arch advancement without a patch minimizes arch reinterventions. Ann Thorac Surg. 2006;82:1577-83. 1574.

21. Nelson W. Applied life data analysis. New York: John Wiley; 1982.

22. Kalbfleisch JD, Prentice RL. The statistical analysis of failure time data. Hoboken: John Wiley \& Sons, Inc.; 2002.

23. Rubin D. Multiple imputation for non-response in surveys. New York: Wiley; 1997

24. Breiman L. Bagging predictors. Machine Learning. 1996;24:123-40.

25. Anderson PK, Borgan O, Gill RD, Keiding N. Statistical models based on counting processes. New York: Springer Verlag; 1995.

\section{Discussion}

Dr Charles D. Fraser (Houston, Tex). Dr Jegatheeswaran and colleagues have really conducted another elegant study from the Congenital Heart Surgeons' Society. I also think it is important to acknowledge that Anusha Jegatheeswaran is the John Kirklin/ David Ashburn Fellow at the Congenital Heart Surgeons' Society Data Center and is due to complete her $\mathrm{PhD}$ in a year and did a wonderful job. I also appreciate her spending a lot of time with me reviewing these very complicated statistics in advance of this presentation.

This study builds on previous work that examined the outcomes in patients with an interrupted aortic arch. The novel aspects of the present study include the evaluation of factors leading to, and the effect of, recurrent arch and left ventricular outflow tract (LVOT) obstruction. Specifically, the study examines time-related rates of, and associated factors for, subsequent arch and LVOT procedures after the initial interrupted aortic arch repair, in addition to examining mortality.

The results are somewhat disappointing, yet likely confirmatory, of what I expect most surgeons believe, and that is, that surgically repaired interrupted aortic arch is a chronic disease with the need for long-term follow-up.

Without further recapitulating the data, I will again summarize, as I understand it, the paper's principal findings. One, multiple procedures after the index operation are common. Second, the risk of subsequent arch procedures decreased after each subsequent arch procedure and the chronic risk of LVOT procedures increased after each subsequent procedure. Third, the factors associated with subsequent arch procedures were related to previous procedures, as well as the characteristics of the anatomy and of the index repair. Of interest, the acute risk of subsequent arch procedure was greatest if the most proximate arch procedure was a catheter intervention. Finally, the need for subsequent procedures after the index operations adversely affects survival.

I would again like to congratulate you on this presentation, and I am left with several questions of practical significance.

What have we learned from this study that will be of value to current practice, specifically what implications did this study bring in terms of the conduct of the primary operation, a better way to repair the arch, a more aggressive stance to deal with the left ventricular outflow tract, or others? 
Dr Jegatheeswaran. Thank you very much for your comments and your question, Dr Fraser.

With respect to the aortic arch, we really have not definitively determined the best procedure, because it depends on the patient's individual characteristics and circumstances, some of which were measured and some of which remain unknown. To definitively address issues of therapy, a randomized, controlled trial, is required, which is particularly difficult in a pediatric population with a rare disease, and can only address one issue at a time. However, from the literature, we know that single centers such as the Texas Children's Hospital in Houston and the Riley Hospital for Children in Indianapolis, have demonstrated excellent results with a dedicated team using only one type of approach, whether that be a direct or staged repair. These observational studies do not, however, tell us which technique is better, they only tell us that either can achieve good results.

With regard to the LVOT, the nature of the lesion is multifaceted. A reluctance to address lesions or an inability to predict the progression of lesions at the index procedure might explain the increasing risk of additional subsequent procedures. It is possible that a more definitive procedure performed early might reduce the risk subsequent procedures for the LVOT.

Regarding catheter-based procedures, those directed at the arch were shown to be less durable than those performed surgically. For the LVOT, catheter-based procedures were directed primarily at the aortic valve, which does not usually provide definitive treatment. Catheter-based procedures directed at subaortic obstruction have not been shown to be of long-term benefit. Nonetheless, however, we still might be tempted, as surgeons, to offer patients catheter-based procedures in the hope of avoiding or delaying more invasive procedures.

Dr Fraser. I think you have answered this in part already, but how should we use these data to counsel parents and patients?

Dr Jegatheeswaran. The most important thing that parents and patients should be counseled about is that the interrupted aortic arch could be a chronic disease. Patients will need long-term follow-up and might require multiple subsequent procedures. In addition, that with each successive procedure, overall mortality is increased. In discussions with patients during follow-up encounters, counseling should emphasize the need for ongoing cardiology follow-up and arrangements for appropriate transition to adult care. Genetic counseling should be included for those diagnosed with DiGeorge syndrome or chromosome 22q11 deletion syndrome.

Finally, based on the results of our study, parents and patients should be encouraged by our contemporary abilities to perform both the primary repair, as well as the subsequent procedures, in addition to the evolution of catheter-based techniques, which are constantly improving.

Dr Fraser. Then, finally, I realize that you were limited in time in the presentation and that prohibited complete elucidation of the demographic, morphologic, and procedural predictors of arch and LVOT reintervention and mortality, but could you share some of those data with us.

Dr Jegatheeswaran. The factors that were not directly reported were those that were thought to be congruent with previous studies.

For subsequent arch procedures, these included a younger age at the index repair; whether a patient was born earlier in the study period; the presence of associated cardiac anomalies; whether the index repair included LVOT repair; whether the left subclavian or a Gore-Tex interposition graft was used to repair the arch at the index procedure; and whether the ventricular septal defect was left open at the index repair, possibly indicating a staged procedure.

Regarding the LVOT, the presence of a small- or medium-size ventricular septal defect; the presence of an anomalous right subclavian artery; and the use of a polytetrafluroethylene graft or a pulmonary homograft for index arch repair increased the risk of a subsequent LVOT procedure.

Regarding mortality, the risk factors included being born earlier in the study period, male gender, and the presence of a small- or medium-size ventricular septal defect, associated cardiac anomalies, or left-sided hypoplasia. Additional factors increasing the risk of mortality that were present at the index arch repair included lower weight, an approach by way of a median sternotomy, and placement of a pulmonary artery band or a systemic-to-pulmonary arterial shunt.

Dr Osman O. Al-Radi (Toronto, Ontario, Canada). I would like to thank you again for the long hours you spent on this study and for the excellent presentation.

The question is: Did you study whether the risk of the LVOT procedure changed depending on the nature of that procedure, whether it was fibromuscular resection versus a more aggressive procedure such as a Konno? In other words, did the type of the first LVOT procedure affect the risk of subsequent LVOT procedures?

Dr Jegatheeswaran. Thank you, Dr Al-Radi.

For subsequent LVOT procedures, it was noted that the type and timing of the subsequent procedures were not significantly associated with an increased risk, although the numbers and statistical power were likely not adequate to be definitive.

Dr Francois Lacour-Gayet (New York, NY). I really enjoyed your paper.

From a practical surgical standpoint, it is important to understand what is, as you said, the optimal first operation to perform. Also, there are clearly different types of patients, those with a very small aortic annulus and the risk of important LVOT obstruction in the future, another where the aortic annulus is acceptable. We were talking about a type A compared with a type B obviously.

You are showing kind of alarming but classic information about the risk of a secondary LVOT obstruction. Actually, in a series in Paris 12 years ago, we found that $30 \%$ of the patients with an interrupted aortic arch required a secondary LVOT procedure.

I have 2 questions. Can you try to be more specific to tell us what was the first procedure that was most frequently performed in your series, namely, was it a subaortic membrane resection? For the second procedure, what was the procedure, was it a second resection, or was it a Ross-Konno operation?

Dr Jegatheeswaran. For LVOT procedures performed concomitantly with index arch repair, fibromuscular resection was the most common procedure type. For LVOT procedures performed after the index arch repair, catheter-based aortic valve dilations were the most common, in addition to fibromuscular resection.

Dr Lacour-Gayet. My final question is that knowing this and knowing that there is a risk of nearly $35 \%$, or even more, of late 
subaortic obstruction, why not start by doing first an operation that is going to fix it, namely, a neonatal Ross-Konno? Also, I understand this is not exactly a question for you, but this is an idea I just want to send out.

My personal practice has been when the aortic annulus is real small and a type $2 \mathrm{~B}$, to go on and do a neonatal Ross. We do no$\mathrm{t}$ have a long series, but the results seem to be satisfactory; of course, with the problem, I think in a Ross-Konno, the problem that we have with the LVOT reconstruction that will require also reoperation.

Dr Jegatheeswaran. Thank you for your comment. The current study lacked sufficient quantitative anatomic detail to allow the prediction of which patients might be expected to develop or manifest important LVOT obstruction after index repair, for whom procedures to address this at the index repair might prevent the need for subsequent procedures. This is a question that remains suboptimally answered.

Dr Giovanni Stellin (Padova, Italy). I wonder whether you found any difference of incidence of LVOT obstruction among the 3 different anatomic types (A, B, C).

Dr Jegatheeswaran. For all risk analyses, an association with anatomical type of IAA was explored, but was not statistically significant for either subsequent arch or subsequent left ventricular outflow tract procedures. 
APPENDIX 1. Participating Congenital Heart Surgeons' Society institutions

\begin{tabular}{l} 
Institution name \\
\hline United States \\
University of Alabama at Birmingham, Birmingham, Alabama \\
The Children's Hospital, Denver, Colorado \\
Miami Children's Hospital, Miami, Florida \\
University of Miami, Miami, Florida \\
All Children's Hospital, St. Petersburg, Florida \\
Loma Linda University Medical Center, Loma Linda, California \\
Children's Hospital of Los Angeles, Los Angeles, California \\
University of California, Los Angeles, School of Medicine, Center for \\
Health Science, Los Angeles, California \\
Children's Hospital and Health Center, San Diego, California \\
University of California, San Francisco, California \\
Children's Memorial Hospital, Chicago, Illinois \\
University of Chicago, Chicago, Illinois \\
University of Iowa Hospitals and Clinics, Iowa City, Iowa \\
The Children's Hospital, Boston, Massachusetts \\
Mott Hospital, Ann Arbor, Michigan \\
Children's Hospital of Michigan, Detroit, Michigan \\
Mayo Clinic, Rochester, Minneapolis \\
University of Nebraska, Nebraska, Nevada \\
Children's Hospital of Buffalo, Buffalo, New York \\
Columbia Presbyterian, New York, New York \\
Duke University Medical Center, Durham, North Carolina \\
Children's Hospital Medical Center, Cincinnati, Ohio \\
Milton S. Hershey Medical Center, Hershey, Pennsylvania \\
The Children's Hospital of Philadelphia, Philadelphia, Pennsylvania \\
St. Christopher's Hospital for Children, Philadelphia, Pennsylvania \\
Children's Hospital of Pittsburgh, Pittsburg, Pennsylvania \\
Medical University of South Carolina, Charleston, South Carolina \\
Primary Children's Hospital, Salt Lake City, Utah \\
Canada \\
British Columbia Children's Hospital, Vancouver, British Columbia \\
Hospital for Sick Children, Toronto, Ontario \\
Heal Children's Hospital, Montreal, Quebec \\
Haulo, Brazil \\
\hline
\end{tabular}




\section{APPENDIX 2. Statistical methods}

Flow charts were created to track patients through multiple consecutive procedures to death or the last follow-up visit.

\section{Nested Competing Risks}

Competing risks analyses were used to examine the rates of transition from an initial state (hazard function) to the mutually exclusive time-related events of various procedure types or death without that procedure type. This was used to determine the proportion of patients reaching these events or states at any given time after the initial state.

Competing risks analyses were performed in a similar manner for each of the following mutually exclusive, competing outcomes: (1) from the index IAA repair to either death or a first subsequent arch procedure, (2) from a first subsequent arch procedure to either death or a second subsequent arch procedure, (3) from the index IAA repair to either death or a first subsequent LVOT procedure (still at risk or no longer at risk of subsequent LVOT procedures), (4) from a first subsequent LVOT procedure to either death or a second subsequent LVOT procedure. Patients considered no longer at risk of LVOT procedures, underwent repairs such as the Damus-Kaye-Stansel or heart transplantation, and were censored at that time. This was also done within the modulated renewal context (see below). For each competing risks analysis, non-risk-adjusted nonparametric estimates for time-related freedom from death or the specified procedure type were plotted using the Kaplan-Meier method. The underlying hazard function was modeled parametrically, determining multiple phases of risk, as previously described. ${ }^{3}$ All graphs were truncated when approximately $10 \%$ of patients remained at risk.

\section{Modulated Renewal}

Repeated arch and LVOT procedures were analyzed as time-related repeating events with the unit of study being the patient and not the procedure (arch or LVOT). Nelson's cumulative event method provided nonparametric estimates, ${ }^{21}$ and a multiphase hazard method provided the parametric estimates. ${ }^{3}$ Because the temporal pattern of risk for each additional subsequent event was similar, we used the modulated renewal process method. ${ }^{22}$ For this, the patients experiencing a first event were restarted at a new time zero and tracked to a second event, and so forth, for each successive event. ${ }^{22}$

\section{Risk Analysis}

We used multiple imputation ${ }^{23}$ using the Markov Chain Monte Carlo technique to impute the missing values. We used fivefold multiple imputation using the Statistical Analysis Systems procedure PROC MI, version 9.2 (SAS Institute, Inc, Cary, NC). In multivariate hazard modeling, for each imputed complete data set, we have estimated the regression coefficients and their variance-covariance matrix. Then, using the method of Rubin, ${ }^{23}$ we combined the estimates from the 5 models. This was performed using the SAS procedure PROC MIANALYZE, version 9.2 (SAS Institute). The relevant missing value indicator variables were created and included in multivariate analyses to adjust for possible bias introduced by missing data.

The demographic, morphologic, and procedural factors associated with each outcome were sought through multivariate analysis of these parametric models. Only variables with less than $40 \%$ of data missing, and those associated with more than 5 events were included, to minimize the risk of model overdetermination. For continuous variables, different mathematic transformations were tested for optimal calibration of the relationship to risk (note, for the interval from the index procedure to the most recent procedure this was calculated as $1 /($ variable +1$)$, as the intervals were 0 in some cases), and the significance of various interaction terms was explored. Nine time-varying covariates were created to adjust for the effects of other procedures in our risk analyses. These variables included the length of the interval from the index procedure to the most recent procedure of a given type (arch, LVOT, "other"), specification of the most recent procedure (arch, LVOT, "other"), and the number of cumulative procedures of each type (arch, LVOT, "other"; see the example patient described, which demonstrates how these variables were created). To ensure adjustment for these time-varying covariates, these variables were always included in the bootstrap modeling used to assess for variable entry reliability. For the arch model, all time-varying intervals and specification of the most recent procedure type (LVOT or "other") were always included in the multivariate modeling. For the LVOT model, no time-varying covariates were included because the number of events was less, and the initial attempts at model building with these variables included showed they were not significant. For the mortality model, all time-varying covariates were always included in the modeling, except for the most recent procedure as an arch procedure. An initial bootstrap was performed without mandating inclusion of any specific variable into the modeling to determine which transformation of the interval variables should always be included in the subsequent final model building. Bootstrap bagging was then performed, again with these time-varying covariates always included, and cluster analysis was used to further guide the final variable selection and to assess the reliability of the variable inclusion into the final multivariate models. Missing value indicator variables were entered into the final multivariate models, as appropriate.

The risk factors for subsequent arch and LVOT procedures were initially identified by bootstrap bagging variable selection $^{24}$ using 500 resampled data sets. $P=.01$ was used in the automated analysis as variable entry criteria. From the output, variables or clusters of variables appearing in $50 \%$ or more of the bootstrap sample analyses were considered sufficiently reliable for inclusion in the final multivariate model building. 


\section{Factors Associated With Mortality}

The factors associated with mortality were explored within the same modulated renewal context as repeated procedures by handling the event of death as a competing risk. ${ }^{25}$ This allowed us to include occurrences of preceding procedural events and their timing, number, type, and interaction as potential associated factors for death. Nonproportionality of risk was accommodated using the multiphase hazard method. ${ }^{3}$

\section{Example Patient}

The following example patient demonstrates a sample sequence of events for an IAA patient. This patient underwent
3 subsequent procedures after the index procedure. Each procedure was given 1 record in the data set, resulting in 4 records, and the time-varying covariate variables change for each record. The first set of 3 variables denoted the most recent procedure type (pr_aar, pr_lvot, pr_oth). The next set of 3 variables denoted the cumulate number of each type of procedure (cum_aar, cum_lvot, cum_oth). The final set of 3 variables denoted the interval from the index procedure to the most recent procedure of that type in years (iv_aar, iv_lvot, iv_oth). As this example is followed through the 4 procedures, the adjustment in the timevarying covariates can be seen.

\section{Example Patient}

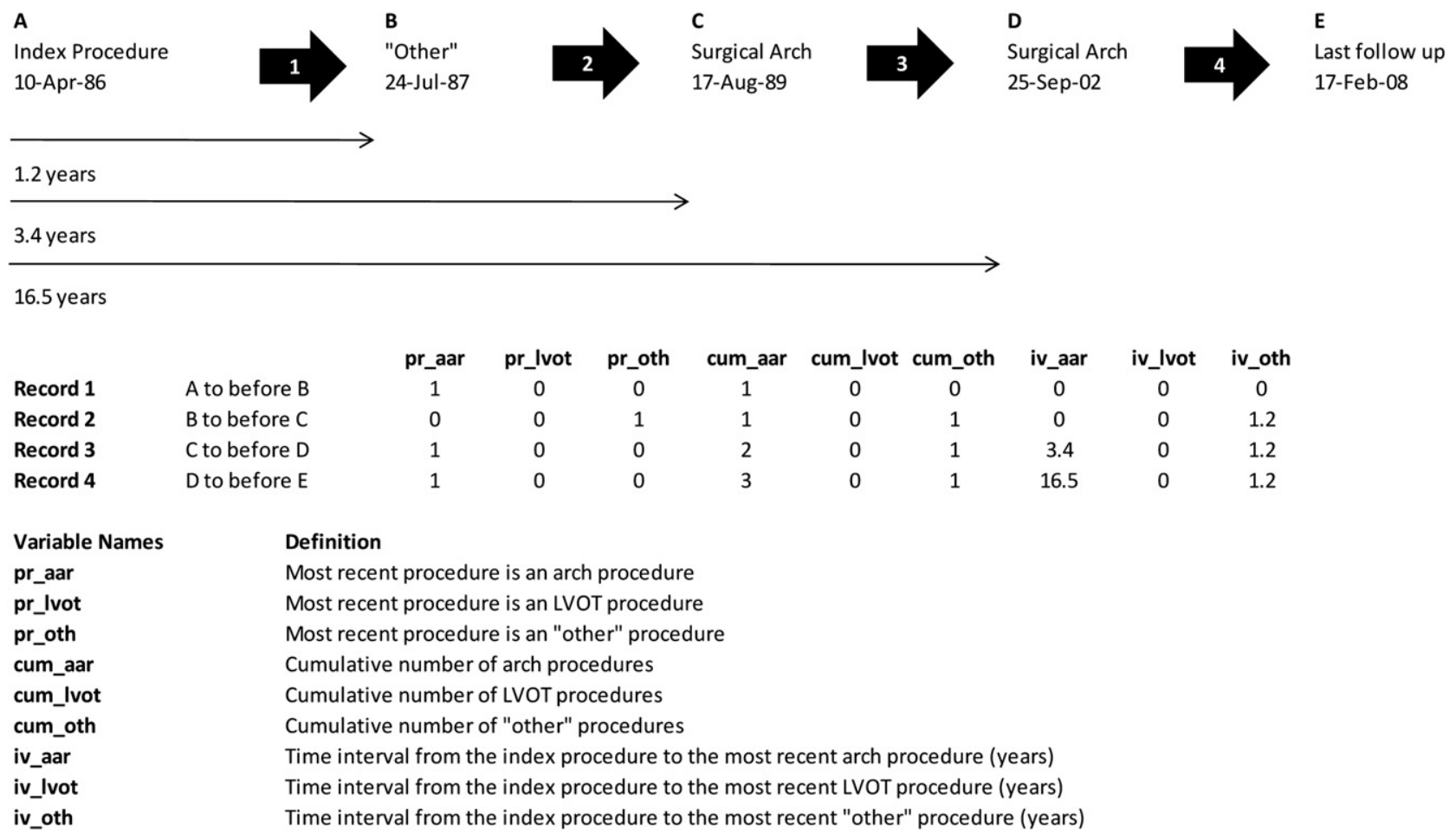


APPENDIX 3. Type of subsequent aortic arch, left ventricular outflow tract, and "other" procedures stratified by subsequent procedure number

\begin{tabular}{lr}
\hline \multicolumn{1}{c}{ A. Type of aortic arch procedure } & 1 \\
\hline Balloon dilation of aortic arch & 54 \\
Patch augmentation of aortic arch & 38 \\
End to end anastomosis & 5 \\
Aorto-aortic bypass & 10 \\
Replace aorto-aortic bypass & 5 \\
Aortic interposition graft & 7 \\
Total & $\mathbf{1 1 9}$ \\
\hline
\end{tabular}

\begin{tabular}{l} 
B. Type of LVOT procedure \\
\hline Balloon dilation of aortic valve \\
Balloon dilation of aortic and subaortic region \\
Balloon dilation of subaortic region \\
Fibromuscular resection \\
Fibromuscular resection, aortic valvuloplasty \\
Aortic valvuloplasty \\
Konno procedure \\
Modified Konno, aortic valvuloplasty \\
Ross/Konno procedure \\
Left ventricle to aorta conduit \\
Rastelli procedure \\
Truncal valvuloplasty \\
Damus-Kaye-Stansel procedure \\
Mechanical aortic valve replacement
\end{tabular}

1
12
3
1
34
2
2
6

2
1
3
1
2

Aortic annular enlargement, mechanical valve replacement,

$$
\text { fibromuscular resection }
$$

\begin{tabular}{l} 
Total \\
C. Type of surgical "other" procedure \\
\hline
\end{tabular}

Heart transplant

Arterial switch, ASD/PFO, VSD closure

Double switch

Atrial switch, deband PA, VSD closure

Atrial switch, Rastelli

Rastelli procedure

Damus-Kaye-Stansel

Glenn procedure

Adjust modified Glenn

Hemi-Fontan

Hemi-Fontan, deband PA, atrial septectomy

Fontan

Pulmonary conduit

Pulmonary conduit, VSD closure

Right ventricular outflow tract patch, muscle bundle resection, deband PA

Pulmonary conduit reoperation \pm ASD/PFO closure \pm PA procedure \pm repair RVOT pseudoaneurysm

ASD/PFO closure

VSD + ASD closure

VSD closure + ASD closure, deband PA,

PA procedure

VSD closure \pm PA procedure \pm ligation of innominate artery

$\mathrm{VSD}+\mathrm{ASD}$ closure, left ventricle to right atrial patch

69

2
8
12
1
4
5
30

$\begin{array}{llcr}\mathbf{3} & \mathbf{4} & \geq \mathbf{5} & \text { Total } \\ 2 & 1 & 2 & \mathbf{6 7} \\ 3 & & & \mathbf{5 3}\end{array}$

1

53

6

\begin{tabular}{lllll}
2 & 3 & 2 & 158 \\
\hline & 3 & 4 & $\geq 5$ & Total
\end{tabular}

2

tal 


\begin{tabular}{|c|c|c|c|c|c|c|}
\hline C. Type of surgical "other" procedure & 1 & 2 & 3 & 4 & $\geq \mathbf{5}$ & Total \\
\hline PA band or revision of PA band & 5 & & & & & 5 \\
\hline Deband PA, patch main PA & 1 & & & & & 1 \\
\hline Systemic to PA shunt & 2 & & & & & 2 \\
\hline Systemic to PA shunt revision or replacement & & 1 & 1 & & & 2 \\
\hline Aortopulmonary window repair & 4 & & & & & 4 \\
\hline Release bronchial compression & 2 & 1 & & & & 3 \\
\hline Right ventricular outflow tract patch & & & & & 1 & 1 \\
\hline Tricuspid valve repair & & 1 & & & & 1 \\
\hline Tricuspid valve repair, PFO, VSD closure & 1 & & & & & 1 \\
\hline Ligation of main pulmonary artery & & & & & 1 & 1 \\
\hline Remove thrombus in right atrium & & 1 & & & & 1 \\
\hline Replace VSD patch & 1 & & & & & 1 \\
\hline Other & 3 & & & & & 3 \\
\hline Total & 103 & 25 & 5 & $\mathbf{0}$ & 5 & 138 \\
\hline D. Type of catheter-based "other" procedure & 1 & 2 & 3 & 4 & $\geq \mathbf{5}$ & Total \\
\hline Balloon conduit and/or pulmonary artery(ies) & 8 & 5 & 2 & 3 & & 18 \\
\hline Balloon aorta-left pulmonary artery shunt & 1 & & & & & 1 \\
\hline Balloon superior vena cava & & & 1 & & & 1 \\
\hline Balloon and stent conduit and/or pulmonary artery & 8 & 4 & 1 & 2 & 4 & 19 \\
\hline Balloon pulmonary artery and stent innominate artery & & 1 & & & & 1 \\
\hline Balloon and stent innominate artery & 1 & & & & & 1 \\
\hline Coil occlusion of collateral & & 2 & 1 & 1 & 3 & 7 \\
\hline Balloon of pulmonary artery and coil occlusion of collateral artery & 1 & & & & & 1 \\
\hline Balloon and blade septostomy of atrial septum & 1 & & & & & 1 \\
\hline Endocardial biopsy after transplantation & 1 & 2 & 1 & & & 4 \\
\hline Total & 21 & 14 & 6 & 6 & 7 & 54 \\
\hline
\end{tabular}

$A S D$, Atrial septal defect; $P F O$, patent foramen ovale; $V S D$, ventricular septal defect; $P A$, pulmonary artery; $R V O T$, right ventricular outflow tract. 
APPENDIX 4. Final multivariate model for subsequent aortic arch procedures, LVOT procedures, and mortality after index procedure

\section{Variable}

Subsequent aortic arch procedures

Early phase

Interval from index procedure to most recent arch procedure (y) (inverse transformation)

Most recent procedure an LVOT procedure

Interval from index procedure to most recent LVOT procedure (y)

Most recent procedure an "other" procedure Interval from index procedure to most recent "other" procedure (y)

Presence of aortopulmonary window

Age at of index procedure (y) (inverse transformation)

Index procedure included concomitant LVOT procedure

Left subclavian artery used to repair aortic arch during index procedure

VSD closed during index procedure

Most recent procedure a surgical arch procedure

Late phase

Interval from index procedure to most recent arch procedure (y) (inverse transformation)

Most recent procedure an LVOT procedure

Interval from index procedure to most recent LVOT procedure (y) (inverse transformation)

Most recent procedure an "other" procedure

Interval from index procedure to most recent "other" procedure (y) (inverse transformation)

Presence of truncus arteriosus

Date of birth to study enrollment start date (y) (natural log transformation)

PTFE interposition graft used to repair aortic arch during index procedure

Cumulative number of arch procedures

Subsequent LVOT procedures

Early phase

Presence of anomalous right subclavian artery

Homograft pulmonary artery used to repair aortic arch during index procedure

Most recent procedure is index procedure

$\underline{\text { Late phase }}$

VSD of small or medium size

PTFE interposition graft used to repair aortic arch during index procedure

\section{Mortality}

\section{Early phase}

Cumulative number of arch procedures

Interval from index procedure to most recent arch procedure (y) (natural log transformation)

Most recent procedure an LVOT procedure

Cumulative number of LVOT procedures

Interval from index procedure to most recent LVOT procedure (y)

Most recent procedure an "other" procedure

Cumulative number of "other" procedures

Interval from index procedure to most recent "other"
Estimate \pm SE

$P$ value

Reliability*

\begin{tabular}{|c|c|}
\hline $13 \pm 4.1$ & .002 \\
\hline$-2.8 \pm 1.1$ & .02 \\
\hline $0.82 \pm 0.25$ & .001 \\
\hline$-5.1 \pm 1.4$ & .0002 \\
\hline $0.69 \pm 0.25$ & .006 \\
\hline $0.77 \pm 0.36$ & .03 \\
\hline $0.22 \pm 0.07$ & .001 \\
\hline $3.1 \pm 1.2$ & .01 \\
\hline $1.4 \pm 0.45$ & .002 \\
\hline$-0.61 \pm 0.25$ & .01 \\
\hline$-2.8 \pm 0.87$ & .002 \\
\hline
\end{tabular}

$2.5 \pm 0.70$

.0003

$-1.7 \pm 0.63 \quad .008$

$-3.3 \pm 0.77<.0001$

$1.4 \pm 0.44 \quad .0009$

$2.3 \pm 0.69 \quad .001$

$2.0 \pm 0.44<.0001 \quad 67 \%$

$-0.51 \pm 0.13 \quad<.0001<72 \%$

$\begin{array}{lll}1.8 \pm 0.31<.0001 & \quad<3 \%\end{array}$

$0.97 \pm 0.19 \quad<.0001 \quad 77 \%$

$0.88 \pm 0.34 \quad .01 \quad 76 \%$

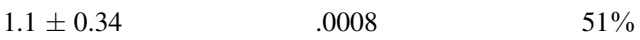

$\begin{array}{ll}0.86 \pm 0.40 \quad .03 & 50 \%\end{array}$

$\begin{array}{lll}1.2 \pm 0.39 & .002 & 70 \%\end{array}$

$0.98 \pm 0.43 \quad .02 \quad 60 \%$

$\begin{array}{rc}1.5 \pm 0.27 & <.0001 \\ -1.3 \pm 0.58 & .03 \\ 0.48 \pm 0.48 & .3 \\ 0.34 \pm 0.43 & .4 \\ 0.28 \pm 0.11 & .01 \\ -0.22 \pm 0.44 & .6 \\ 1.1 \pm 0.23 & <.0001 \\ -0.59 \pm 0.26 & .02\end{array}$
procedure $(\mathrm{y})$ 


\section{Variable}

Variables related to morphology

Male

Presence of truncus arteriosus

Date of birth to study enrollment start date (y)

VSD of small or medium size

Hypoplastic left heart class I

Variables related to index IAA repair

Weight at index procedure $(\mathrm{kg})$ (inverse transformation)

Index procedure done via thoracotomy

Pulmonary artery banding procedure done at index procedure

Systemic to PA to arterial shunt created during index procedure

Variables related to procedures after index IAA repair

Subsequent VSD closure

Subsequent procedure with total circulatory arrest

Subsequent aortic procedure with patch augmentation
Estimate \pm SE

$P$ value

Reliability*

$-0.47 \pm 0.16$

.004

$<.0001$

$<.0001$

.03

.0001

.006

$<.0001$

.0003

.002

$0.73 \pm 0.24$

.0009

$<.0001$

$67 \%$

$1.2 \pm 0.35$

$1.4 \pm 0.33$

.01

$69 \%$

$80 \%$

$65 \%$

$62 \%$

$57 \%$

$72 \%$

$50 \%$

$54 \%$

$79 \%$

$-1.4 \pm 0.56$
$82 \%$

$62 \%$

LVOT, Left ventricular outflow tract; VSD, ventricular septal defect; PTFE, polytetrafluoroethylene; IAA, interrupted aortic arch; PA, pulmonary artery. *Variables without reliability estimates were always included in models as time-varying covariate adjustment factors.

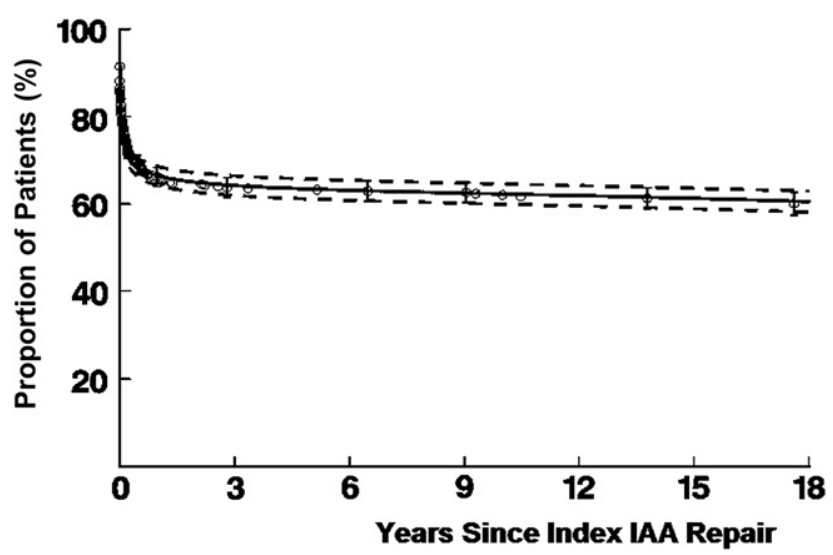

APPENDIX 5. Overall time-related survival of 447 neonates since index IAA repair. All patients began at the time of index IAA repair at a CHSS member institution. Solid lines represent parametric point estimates; dashed lines enclose $70 \%$ confidence intervals (CI); circles with error bars represent nonparametric estimates. The overall survival at 1, 3, 6 , and 9 years was $66 \%, 64 \%, 63 \%$, and $62 \%$, respectively. 


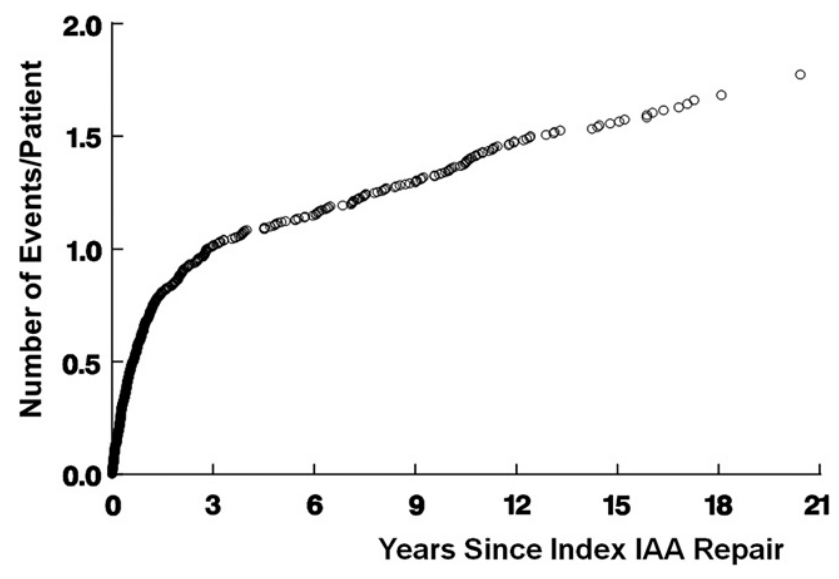

FIGURE E1. Cumulative hazard for subsequent procedures of any type. This graph demonstrates the cumulative number of events per patient at any given point since the index procedure. Circles represent any subsequent procedure $(n=436)$. 


\section{Month}
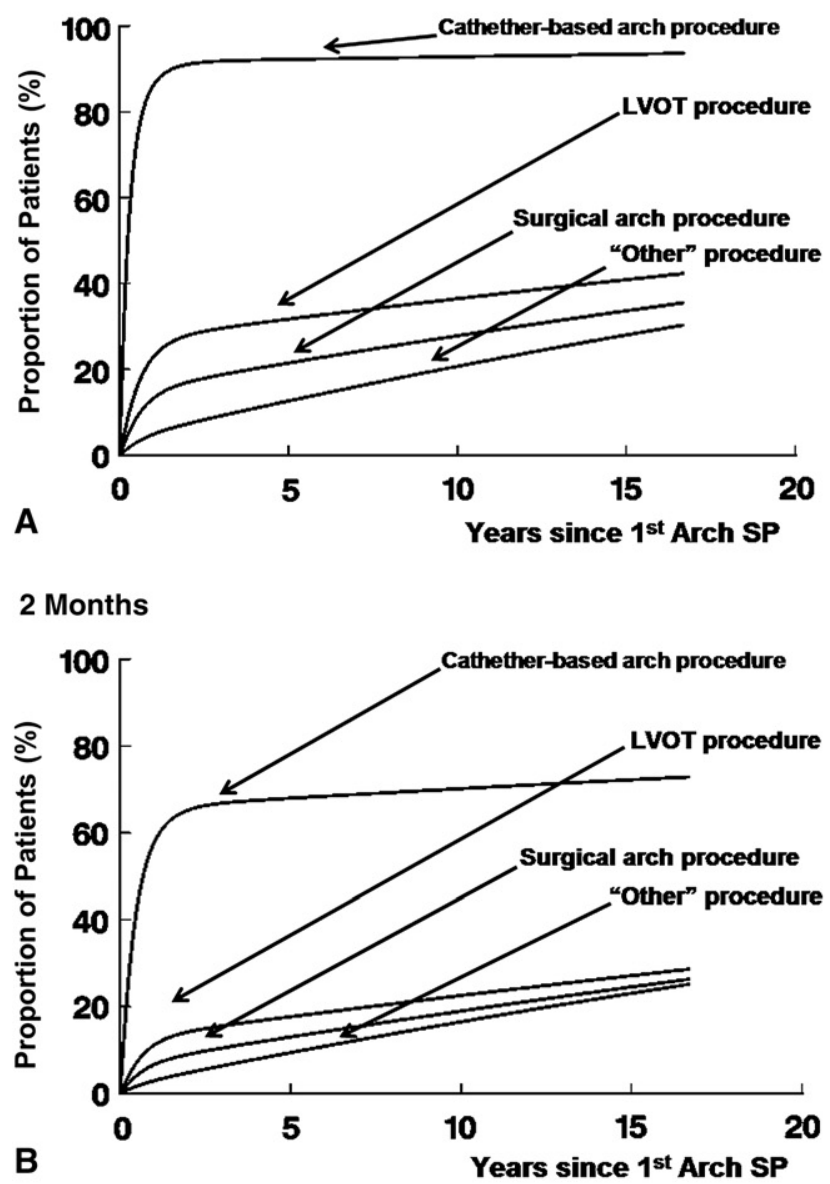

\section{Months}

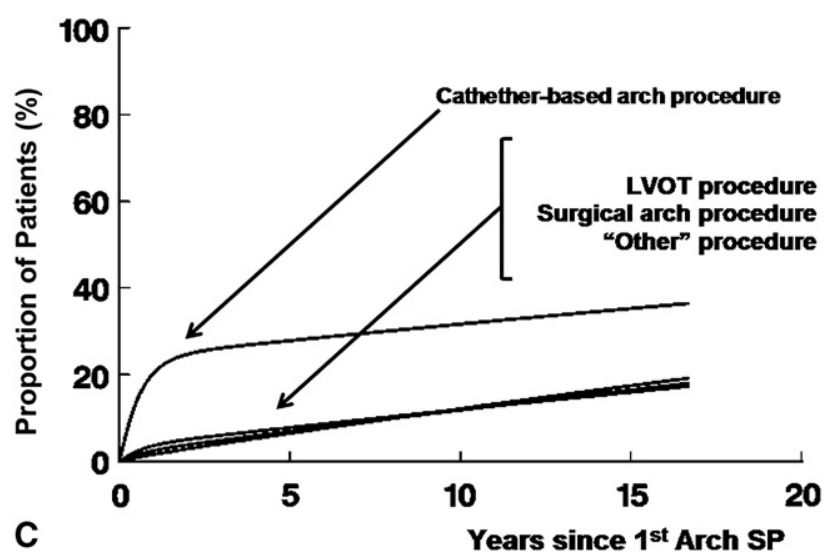

FIGURE E2. Risk of a second subsequent arch procedure stratified by type of most recent procedure (catheter-based arch, "other," surgical arch, LVOT procedure) and interval (1 month, 2 months, and 4 months) from the index procedure to the most recent arch procedure (in this case the first subsequent arch procedure) for a patient with a particular risk profile. This graph serves to illustrate "risks related to previous procedures" 1 and $4(A-D)$, Table 2, for subsequent arch procedure. A "typical" patient profile was assumed (ie, one who had IAA without an additional cardiac diagnosis, a birth date near the middle of the study era, an index repair at an average age for patients in the second renewal, an index repair without concomitant LVOT resection, without the use of polytetrafluroethylene or subclavian artery for arch repair, and without concomitant VSD closure, and 1 subsequent arch procedure). These 3 graphs demonstrate that as the interval from the index procedure to the most recent arch procedure (in this case, the first subsequent arch procedure) increases (from 1 to 2 to 4 months), the risk of a second subsequent arch procedure decreases, independent of what the most recent procedure had been. This finding is tantamount to risk 1 in Table 2. Furthermore, the risk of a second subsequent arch procedure is generally greatest when the most recent procedure was a catheter-based arch procedure, followed by an LVOT procedure, a surgical arch procedure, and an "other" procedure. This finding illustrates the complex, timedependent interrelationships among the subsequent procedures (also shown in Table 2). The exact order of risk of the 4 types of most recent procedures differs between Table 2 and $\mathrm{A}, \mathrm{B}$, and $\mathrm{C}$ because the former risks were calculated separately for the acute and chronic risk phases, and those in A, B, and $\mathrm{C}$ were calculated as a composite risk. Solid lines represent parametric point estimates. Arch, Aortic arch; SP, subsequent procedure. 